\title{
ES LA HORA DEL MARKETING INTERNO
}

\author{
Mª Isabel Sánchez Hernández \\ Francisco Javier Miranda González \\ Universidad de Extremadura \\ isanchez@unex.es \\ fmiranda@unex.es
}

https://doi.org/10.17979/redma.2009.01.02.4683

\section{Mª Isabel Sánchez Hernández}

Doctora en Ciencias Económicas y Empresariales por la Universidad de Extremadura. MSc in Economics por la University of Aberdeen (U.K), Master en Organización y Dirección de Recursos Humanos por la Universidad Politécnica de Madrid y Licenciada en Ciencias Económicas y Empresariales por la Universidad Autónoma de Madrid. Actualmente es profesora colaboradora del Área de Organización de Empresas. Su actividad académico-profesional radica en la conjugación de la disciplina del Marketing aplicado a la gestión de intangibles, recursos humanos, cultura, reputación y responsabilidad social corporativa.

Universidad de Extremadura

Facultad de Ciencias Económicas y Empresariales

Departamento de Dirección de Empresas y Sociología

Avenida de Elvas $\mathrm{s} / \mathrm{n}$ - 06071 Badajoz

924289000 ext.89149 y 86881

\section{Francisco J. Miranda González}

Doctor en Ciencias Económicas y Empresariales por la Universidad de Extremadura y Master en Gestión de la Producción, la Calidad y la Tecnología por la Universidad Politécnica de Madrid. Profesor Titular de Universidad del Área de Organización de Empresas. Es autor de varios libros y publicaciones en revistas internacionales. En la actualidad sus principales líneas de investigación son la gestión de la calidad en servicios; el diseño y desarrollo de nuevos productos y servicios; internet como herramienta empresarial y el comercio electrónico B2B. 


\section{Resumen}

Convencidos de que el capital humano es la principal fuente de ventajas competitivas en los mercados (Penrose, 1959), y teniendo en cuenta que la literatura académica que analiza qué tipo de factores determinan el éxito del proceso de desarrollo de un nuevo servicio no considera directamente la aplicación de programas de marketing interno, el objetivo de este trabajo ha sido descubrir su relevancia relativa en este campo de investigación.

Para conseguir este objetivo, primero se han recopilado los artículos relacionados con innovación en servicios en las revistas internacionales indexadas en el JCR (Journal Citation Report). En segundo lugar, se han seleccionado los artículos centrados en factores de éxito para finalmente, clasificar los diferentes factores hallados en cinco grandes grupos siguiendo las categorías de De Brentani (1995) y Johnson et al. (2000).

Como conclusión, se ha constatado que el tópico general de marketing interno, alguno de sus instrumentos o bien su base filosófica (como la orientación al mercado), están siendo considerados un factor emergente de éxito en los procesos de desarrollo de nuevos servicios, capaz de llegar a ser el centro de atención en un futuro cercano.

\section{Palabras clave}

Marketing Interno, Desarrollo de Nuevos Servicios, Factores de Éxito

\section{Abstract}

IT'S TIME FOR INTERNAL MARKETING

Convinced that human capital is the most important source of competitive advantage in markets (Penrose, 1959), and bearing in mind that academic literature advocate to 
analyse which kind of factors determine success in new service development process do not consider directly the application of internal marketing programmes, the aim of this paper is discover its relative relevance in this research area.

To achieve this objective, we have considered the following stages. Firstly, we have recollected papers in international journals included in JCR (Journal Citation Report) index, related to innovation in services. Secondly, we have selected papers devoted to success factors. Finally, we have classified the different factors discovered into five main groups following the De Brentani (1995) and Johnson et al. (2000) categories. Once the general topic of internal marketing or related instruments (like internal communication) or philosophical background (like market orientation) appeared, we have noticed.

As conclusion, we observe that internal marketing considerations are in fact treated as an emergent factor of success in new service development process able to become the centre of attention in the nearest future.

\section{Key Words}

Internal Marketing, New Service Development, Success Factors

\section{Resumo}

Cientes de que o capital humano é a principal fonte de vantagens competitivas nos mercados (Penrose, 1959), e tendo em conta que a literatura acadêmica que analisa quais são os fatores que determinam o sucesso do processo de desenvolvimento de um novo serviço não considera diretamente a aplicação de programas de marketing interno, o objetivo deste trabalho tem sido a descoberta da sua importância relativa nesta área de pesquisa.

Para conseguir este objetivo, primeiro tem-se recopilado os artigos relativos a inovação em serviços nas revistas internacionais indexadas no JCR (Journal Citation Report). Em segundo lugar, selecionaram-se os artigos baseados em fatores de 
sucesso e finalmente, clasificaram-se os diferentes fatores em cinco grandes grupos seguindo as categorias de De Brentani (1995) e Johnson et al. (2000).

Como conclusão, constata-se que o tópico geral do marketing interno, algum dos seus instrumentos ou a sua base filosófica (como a orientação ao mercado), estão a ser consideradas um fator emergente de sucesso nos precessos de desenvolvimento de novos serviços, com a capacidade de chegar a ser o centro de atenção no futuro prossimo.

\section{Palabras chave}

Marketing Interno, Desenvolvimento de Novos Serviços, Fatores de Sucesso. 


\section{ES LA HORA DEL MARKETING INTERNO}

"El Marketing Interno es el esfuerzo planificado de motivar a los empleados a través de las técnicas del marketing para implantar e integrar estrategias empresariales de orientación al cliente".

Ahmed y Rafiq (2002, p.9)

La evolución del sector servicios en las economías occidentales es incuestionable. La terciarización de la economía arroja actualmente valores superiores al $65 \%$ de VAB o de ocupación en la UE (Eurostat, 2007).

Explicando esta evolución y siguiendo a Lovelock (1992), destacamos los cambios en el nivel de regulación de la economía por parte de los Gobiernos, una mayor liberalización de los servicios de las Asociaciones Profesionales, las privatizaciones, la revolución de la tecnología y el espectacular crecimiento de las franquicias. Por su parte Cuadrado (2004) hace hincapié en la importancia del creciente consumo de servicios por parte de los individuos y las familias, una creciente utilización de los servicios externos, la expansión del comercio internacional de servicios y el incremento del papel del Sector Público como oferente de servicios políticoadministrativos y vinculados a la expansión del Estado de Bienestar.

El Índice de la Cifra de Negocios del Sector Servicios (ICSS) ${ }^{1}$ - que mide la evolución a corto plazo de las empresas que operan en cinco subsectores de los servicios: comercio, turismo, transporte, tecnologías de la información y las comunicaciones (TIC) y servicios prestados a las empresas - registró en 2005 en la UE un aumento del 7,6 por ciento respecto al año anterior (Eurostat, 2007). El mayor incremento se dio en los servicios prestados a las empresas con un 11,7 por ciento. El resto de los subsectores también creció, pero menos: entre el 8,3 por ciento de las TIC y el 4,8 por ciento de las actividades turísticas.

\footnotetext{
${ }^{1}$ Los indicadores de la actividad del sector servicios se calculan en todos los países de la UE según lo establecido por la legislación comunitaria, utilizando la misma metodología para facilitar su comparación.
} 
En consonancia con estos datos cabe añadir que la investigación en el campo de la innovación en servicios a las empresas ${ }^{2}$ los revela como una de las principales palancas del cambio y progreso económico (Czarnitzki y Spielkamp, 2003). Y dentro de esta categoría, los servicios intensivos en conocimiento, también conocidos por sus siglas en inglés KIBS (Knowledge Intensive Business Services), juegan un papel importante en los sistemas de innovación porque no sólo trasmiten conocimiento, sino que propician "reingeniería de conocimiento" (Muller y Zenker, 2001). Es decir, primero y a través de sus actividades, estimulan las capacidades de innovación en las empresas clientes generándose un ciclo virtuoso en el que se fomenta la propia innovación interna de los KIBS. En palabras de Czarnitzki y Spielkamp (2003), los KIBS son para otros negocios puentes hacia la innovación por su clara capacidad innovadora, especialmente su alto contenido de conocimiento, creatividad, habilidades de gestión y mercado.

Los datos apuntados justifican el hecho de que en los últimos años, los procesos de innovación y desarrollo de nuevos servicios (DNS) hayan adquirido gran interés tanto en el mundo empresarial como en el ámbito académico. Adicionalmente, el capital humano está protagonizando el nuevo paradigma de la Dirección de Empresas actual, que coloca a las personas en el "centro de la estrategia" (Álvarez, 2006, p.46) por ser consideradas la principal fuente de ventaja competitiva sostenible.

Entendemos además, que el trinomio - capital humano, innovación, éxito - viene especialmente reforzado cuando se aplica al sector servicios porque la innovación está siendo entendida de manera general, más allá de lo estrictamente tecnológico, incluyendo tanto la creación de nuevos servicios para el mercado o nuevos para la empresa, como una forma más eficiente de prestar un servicio ya ofrecido (Gadrey et al., 1995; Gallouj y Weinstein, 1997), donde la influencia del capital humano tiene mayor trascendencia.

\footnotetext{
${ }^{2}$ Se encuentran dentro de la categoría de servicios profesionales la consultoría, los sistemas de información, ingeniería, formación, servicios financieros, los servicios de publicidad, las artes gráficas, los servicios legales, la gestión de proyectos o de materiales, la educación, los servicios de bases de datos, las actividades de marketing y relaciones públicas, la ingeniería industrial y la contabilidad, entre otros.
} 
Es en este contexto, donde el marketing interno, inicialmente enmarcado en el campo del marketing de servicios, aparece en el panorama de la Administración de Empresas como un instrumento de gestión que, asentado teóricamente en una orientación al mercado interno, es capaz de vincular el capital humano a la estrategia competitiva de la organización (Ahmed y Rafiq, 1993; 2002). De hecho, y teniendo como punto de referencia las conclusiones de la American Marketing Association Conference de $1984^{3}$, que enfatizaron expresamente el significado del empleado y de su integración en los procesos de innovación, han surgido algunos trabajos que inciden de manera directa en la importancia del marketing interno (Cooper y De Brentani, 1991; Cooper et al, 1994; Storey y Easingwood, 1993,1996, 1998; Lievens et al., 1999; Lievens y Moenaert, 2000a; Lievens y Moenaert, 2000b) como instrumento que busca el compromiso del personal y ayuda a asegurar la consistencia de la estrategia corporativa y el éxito de los nuevos servicios en el mercado.

Como señala Bourner (1996), existen buenas razones para dedicar esfuerzos a revisar la literatura antes de adentrarse en un campo de investigación. Convencidos de que el capital humano es la principal fuente de ventajas competitivas en los mercados (Penrose, 1959), que el marketing interno se revela como un potente mecanismo de gestión y dado que vivimos en una economía de servicios, hemos profundizado en la literatura académica de impacto que aborda estas relaciones conceptuales que mostramos en la Figura 1.

\section{Figura 1: El Marketing Interno en el Ciclo Virtuoso de las Economías de}

\section{Servicios}

\footnotetext{
${ }^{3}$ George, W. G. y Marshall, C. E. (1984). Developing New Services. American Marketing Association, Proceedings Series.
} 


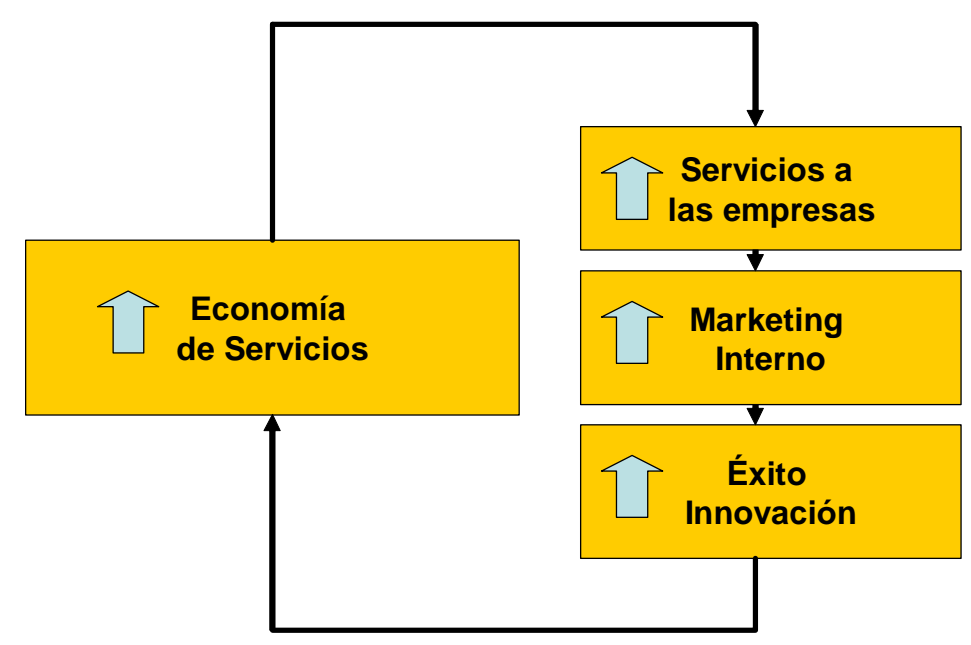

Fuente: Elaboración propia

Con este objetivo, recopilamos todos los artículos relacionados con innovación en servicios, teniendo como base las revistas internacionales indexadas en el JCR (Journal Citation Report). En segundo lugar, seleccionamos los artículos centrados en factores de éxito para después clasificarlos en cinco grandes grupos siguiendo las cuatro categorías tradicionales de De Brentani (1995) y añadiendo la categoría cultural señalada por Johnson et al. (2000).

Tal y como se muestra en la Tabla 1, fruto del análisis exhaustivo de las publicaciones seleccionadas - un total de 21 artículos - se ha podido constatar que el marketing interno, alguno de sus instrumentos o bien la base filosófica que le da consistencia (la orientación al mercado), están siendo considerados como factores emergentes de éxito en la innovación en servicios en dos sentidos:

- Como instrumento de gestión al servicio de la eliminación de barreras a la colaboración interfuncional, potenciando la generación de sinergias organizativas.

- Formando parte de la habilidad en el DNS, potenciando la comunicación interna aumentando efectividad en la gestión del DNS. 
Destacamos el trabajo pionero de Cooper y De Brentani (1991). Su análisis empírico define como factores de éxito dominantes del DNS: las sinergias entre las necesidades del proyecto y los recursos, habilidades y experiencias de la empresa; el ajuste entre el servicio y el mercado; la calidad del proceso de lanzamiento y ejecución que incluye un marketing interno que garantice que el servicio haya sido bien promocionado entre el personal de primera línea; las ventajas del mercado que hacen que un servicio sea designado como único; y por último la calidad en la ejecución.

Y en la misma línea, el análisis de Storey y Easingwood (1993) señalan que el factor más determinante del éxito de un nuevo servicio es el marketing interno y las sinergias que genera, apuntando posteriormente que si se impulsan las actividades de marketing interno, se estará contribuyendo significativamente al resultado de las organizaciones (Storey y Easingwood, 1998). 
Tabla 1: Presencia del Marketing Interno como factor emergente de éxito en el DNS

\begin{tabular}{|c|c|c|c|c|c|c|c|c|c|c|}
\hline $\begin{array}{l}\text { Factores de } \\
\text { Éxito }\end{array}$ & $\begin{array}{l}\text { De Brentani } \\
\text { (1989) }\end{array}$ & $\begin{array}{l}\text { Cooper y De } \\
\text { Brentani (1991) }\end{array}$ & $\begin{array}{l}\text { Martin y } \\
\text { Horne } \\
\text { (1993) }\end{array}$ & $\begin{array}{l}\text { Storey y } \\
\text { Easingwood } \\
\text { (1993) }\end{array}$ & $\begin{array}{l}\text { Cooper et al. } \\
\text { (1994) }\end{array}$ & $\begin{array}{l}\text { Edgett y } \\
\text { Parkinson } \\
\text { (1994) }\end{array}$ & $\begin{array}{l}\text { De } \\
\text { Brentani } \\
\text { (1995) }\end{array}$ & $\begin{array}{l}\text { Martin y } \\
\text { Horne } \\
\text { (1995) }\end{array}$ & $\begin{array}{l}\text { Atuahene- } \\
\text { Gima } \\
(1996)\end{array}$ & $\begin{array}{l}\text { De Brentani } \\
\text { y Ragot } \\
\text { (1996) }\end{array}$ \\
\hline $\begin{array}{l}\text { Naturaleza del } \\
\text { servicio }\end{array}$ & $\begin{array}{l}\text { Innovación } \\
\text { Calidad }\end{array}$ & $\begin{array}{l}\text { Unico/Experto en la } \\
\text { prestación }\end{array}$ & $\begin{array}{l}\text { Ser lider del } \\
\text { mercado }\end{array}$ & - & $\begin{array}{l}\text { Tecnología innovadora. } \\
\text { Ventaja en el servicio al } \\
\text { consumidor. } \\
\text { Responsabilidad }\end{array}$ & - & - & & $\begin{array}{l}\text { Empleados } \\
\text { bien } \\
\text { formados }\end{array}$ & $\begin{array}{l}\text { Innovación (-) } \\
\text { Jovedad para la } \\
\text { organización } \\
\text { Experto en la }\end{array}$ \\
\hline $\begin{array}{l}\text { Características } \\
\text { del producto o } \\
\text { del mercado }\end{array}$ & $\begin{array}{l}\text { Orientación al } \\
\text { mercado } \\
\text { Necesidad del } \\
\text { mercado }\end{array}$ & $\begin{array}{c}\text { Ajuste } \\
\text { Producto/mercado } \\
\text { Tamaño/Crecimiento } \\
\text { de mercado }\end{array}$ & - & $\begin{array}{l}\text { Investigación de } \\
\text { mercado }\end{array}$ & - & $\begin{array}{l}\text { Investigación } \\
\text { de mercado }\end{array}$ & $\begin{array}{l}\text { Necesidades } \\
\text { del mercado }\end{array}$ & - & $\begin{array}{l}\text { Marketing de } \\
\text { relaciones }\end{array}$ & $\begin{array}{l}\text { Tamaño y } \\
\text { Potencial del } \\
\text { Mercado }\end{array}$ \\
\hline $\begin{array}{l}\text { Sinergias del } \\
\text { proyecto }\end{array}$ & $\begin{array}{l}\text { Sinergias de } \\
\text { Marketing }\end{array}$ & $\begin{array}{c}\text { Sinergias. } \\
\text { Marketing interno }\end{array}$ & $\begin{array}{l}\text { Sinergias con } \\
\text { los negocios } \\
\text { de la } \\
\text { organización }\end{array}$ & $\begin{array}{l}\text { Marketing } \\
\text { Interno }\end{array}$ & $\begin{array}{l}\text { Sineraian do Morkating } \\
\text { y de Gestión } \\
\text { Financiera. Marketing } \\
\text { interno }\end{array}$ & $\begin{array}{l}\text { Sinergias con } \\
\text { el mercado }\end{array}$ & $\begin{array}{l}\text { Sinergias con } \\
\text { los recursos } \\
\text { Reputación }\end{array}$ & $\begin{array}{l}\text { Sinergias con } \\
\text { el mercado }\end{array}$ & $\begin{array}{l}\text { (-) Sinergias } \\
\text { tecnológicas }\end{array}$ & $\begin{array}{l}\text { Sinergias con el } \\
\text { mercado }\end{array}$ \\
\hline $\begin{array}{l}\text { La habilidad } \\
\text { en el DNS }\end{array}$ & $\begin{array}{l}\text { Proceso de } \\
\text { DNS }\end{array}$ & $\begin{array}{l}\text { Ejecución: } \\
\text { Lanzamiento } \\
\text { Marketing y } \\
\text { Tecnología. } \\
\text { Marketing interno. } \\
\text { Actividades de } \\
\text { predesarrollo }\end{array}$ & - & $\begin{array}{c}\text { Ventaja } \\
\text { Tecnológica } \\
\text { Correo Directo } \\
\text { Responsabilidad }\end{array}$ & $\begin{array}{c}\text { Comunicaciones. } \\
\text { Preparación del } \\
\text { lanzamiento al } \\
\text { mercado. }\end{array}$ & $\begin{array}{l}\text { Orientación } \\
\text { al mercado } \\
\text { interno } \\
\text { (empleados) } \\
\text { Coordinación } \\
\text { interfuncional }\end{array}$ & $\begin{array}{l}\text { Excelencia } \\
\text { en el proceso } \\
\text { de DNS }\end{array}$ & $\begin{array}{c}\text { Participación } \\
\text { del } \\
\text { concuminor. } \\
\text { Implicación } \\
\text { del gestores } \\
\text { y otros } \\
\text { ompleadoc }\end{array}$ & $\begin{array}{l}\text { Empleados: } \\
\text { fuente de } \\
\text { ideas y } \\
\text { aseguran } \\
\text { lanzamiento } \\
\text { efectivo }\end{array}$ & $\begin{array}{l}\text { Participación del } \\
\text { consumidor } \\
\text { Proceso DNS } \\
\text { formal }\end{array}$ \\
\hline $\begin{array}{l}\text { Cultura de } \\
\text { innovación }\end{array}$ & - & - & - & - & - & $\begin{array}{l}\text { Alto nivel de } \\
\text { compromiso }\end{array}$ & - & - & $\begin{array}{c}\text { Estrategia de } \\
\text { RH orientada } \\
\text { a la } \\
\text { innovación }\end{array}$ & \\
\hline
\end{tabular}

Fuente: Elaboración propia 
Tabla 1: Presencia del Marketing Interno como factor emergente de éxito en el DNS (continuación)

\begin{tabular}{|c|c|c|c|c|c|c|c|c|c|c|c|}
\hline $\begin{array}{c}\text { Factores de } \\
\text { Éxito }\end{array}$ & $\begin{array}{l}\text { Storey y } \\
\text { Easingwood } \\
\text { (1996) }\end{array}$ & $\begin{array}{l}\text { Storey y } \\
\text { Easingwood } \\
\text { (1998) }\end{array}$ & $\begin{array}{l}\text { Lievens et al. } \\
\text { (1999) }\end{array}$ & $\begin{array}{l}\text { Lievens y } \\
\text { Moenaert } \\
(2000 b)\end{array}$ & $\begin{array}{l}\text { Avlonitis } \\
\text { et al. } \\
\text { (2001) }\end{array}$ & $\begin{array}{l}\text { De Brentani } \\
(2001)\end{array}$ & $\begin{array}{l}\text { Johne y } \\
\text { Harborne } \\
\text { (2003) }\end{array}$ & $\begin{array}{l}\text { Blazevic } \\
\text { y Lievens } \\
\text { (2004) }\end{array}$ & $\begin{array}{l}\text { Carvalho } \\
\text { Vieira et } \\
\text { al. (2004) }\end{array}$ & $\begin{array}{l}\text { Matear et } \\
\text { al. (2004) }\end{array}$ & $\begin{array}{l}\text { Van Riel y } \\
\text { Lievens } \\
(2004)\end{array}$ \\
\hline $\begin{array}{c}\text { Naturaleza del } \\
\text { servicio }\end{array}$ & $\begin{array}{l}\text { Calidad } \\
\text { tangible. } \\
\text { Calidad en la } \\
\text { prestación }\end{array}$ & $\begin{array}{c}\text { Calidad } \\
\text { "Servicio } \\
\text { aumentado" } \\
\text { Entrenamiento } \\
\text { de empleados }\end{array}$ & $\begin{array}{l}\text { Intangibilidad, } \\
\text { heterogeneidad, } \\
\text { simultaneidad y } \\
\text { cómo sea de } \\
\text { perecedero el } \\
\text { servicio }\end{array}$ & $\begin{array}{l}\text { Intangibilidad, } \\
\text { heterogeneidad, } \\
\text { simultaneidad y } \\
\text { cómo sea de } \\
\text { perecedero el } \\
\text { servicio }\end{array}$ & $\begin{array}{l}\text { Tipo de } \\
\text { Innovación }\end{array}$ & $\begin{array}{l}\text { Competencias } \\
\text { únicas }\end{array}$ & - & - & $\begin{array}{l}\text { Calidad } \\
\text { global }\end{array}$ & - & - \\
\hline $\begin{array}{c}\text { Características } \\
\text { del producto o } \\
\text { del mercado }\end{array}$ & $\begin{array}{l}\text { Actividades de } \\
\text { Marketing }\end{array}$ & $\begin{array}{l}\text { Conocimiento } \\
\text { del mercado }\end{array}$ & - & - & - & $\begin{array}{l}\text { Tácticas de } \\
\text { Marketing }\end{array}$ & - & - & - & ientacic & \\
\hline $\begin{array}{l}\text { Sinergias del } \\
\text { proyecto }\end{array}$ & $\begin{array}{l}\text { Ajuste del } \\
\text { servicio con la } \\
\text { empresa y con }\end{array}$ & $\begin{array}{c}\text { Marketing } \\
\text { Interno }\end{array}$ & Marketir & Marketing & - & $\begin{array}{c}\text { Sinergias con la } \\
\text { organización }\end{array}$ & $\begin{array}{l}\text { Sinergias } \\
\text { entre los } \\
\text { gestores }\end{array}$ & - & $\begin{array}{l}\text { Sinergias } \\
\text { de } \\
\text { Marketing }\end{array}$ & - & - \\
\hline $\begin{array}{l}\text { La habilidad } \\
\text { en el DNS }\end{array}$ & $\begin{array}{l}\text { Fortaleza en la } \\
\text { distribución. } \\
\text { Comunicación } \\
\text { efectiva }\end{array}$ & $\begin{array}{l}\text { Actividades de } \\
\text { Marketing }\end{array}$ & $\begin{array}{l}\text { pmunicacion } \\
\text { externas e } \\
\text { internas }\end{array}$ & $\begin{array}{l}\text { oxunicacione } \\
\text { externas e } \\
\text { internas }\end{array}$ & $\begin{array}{l}\text { Proceso de } \\
\text { DNS }\end{array}$ & $\begin{array}{l}\text { Proceso formal de } \\
\text { DNS }\end{array}$ & $\begin{array}{c}\text { Comunica- } \\
\text { ción } \\
\text { erfunciona }\end{array}$ & $\begin{array}{l}\text { Comunica- } \\
\text { ción } \\
\text { Aprendizaje } \\
\text { organizativo }\end{array}$ & $\begin{array}{l}\text { Proceso } \\
\text { formal de } \\
\text { DNS }\end{array}$ & - & $\begin{array}{l}\text { Comunicación } \\
\text { Información } \\
\text { de los } \\
\text { gestores }\end{array}$ \\
\hline $\begin{array}{l}\text { Cultura de } \\
\text { innovación }\end{array}$ & - & - & - & $\begin{array}{l}\text { Cultura de } \\
\text { emprendedurismo, } \\
\text { creatividad }\end{array}$ & - & $\begin{array}{l}\text { Cultura de } \\
\text { emprendedurismo, } \\
\text { creatividad }\end{array}$ & & - & $\begin{array}{l}\text { Imagen } \\
\text { corporativa }\end{array}$ & - & $\begin{array}{l}\text { Reducción de } \\
\text { la } \\
\text { incertidumbre }\end{array}$ \\
\hline
\end{tabular}

REDMARKA UIMA-Universidad de A Coruña - CIECID 
A la vista de la revisión llevada a cabo y partiendo de la base de que el éxito se gestiona, no siendo atribuible a la intuición o la suerte, parece claro que es necesario potenciar el marketing interno en la Administración de Empresas. En concreto, nuestro análisis nos revela que:

- El esfuerzo, tanto empresarial como académico, en el campo de la innovación en servicios se revela como prioritario en términos de competitividad.

- En los procesos de desarrollo de nuevos servicios debe prestarse más atención a los elementos organizativos intangibles, como la cultura o la gestión del capital humano, dado que se está demostrando que tienen importantes efectos en el éxito de los mismos.

- Se va concediendo más importancia al marketing interno como instrumento que, en busca del compromiso del capital humano, ayude a asegurar la consistencia de la estrategia corporativa y el éxito de los nuevos servicios en el mercado.

- El marketing interno se revela como un factor emergente de éxito en el DNS que debe ir ganando protagonismo en la gestión empresarial de las organizaciones de servicios que quieran seguir siendo competitivas en sus mercados.

Podemos afirmar entonces, que es la hora del marketing interno. Sin embargo, a pesar del interés que viene suscitando el marketing interno entre los académicos, el hecho de que su aplicación sea todavía muy limitada obedece, según Gounaris (2006, p.434) a la ausencia de una "filosofía de base" que facilite su puesta en funcionamiento. El autor hace referencia a una filosofía corporativa análoga a la orientación al mercado (Kolhi y Jaworski, 1990; Narver y Slater, 1990) que preceda a la habilidad de la compañía para desarrollar estrategias efectivas de marketing interno.

Así, lejos de lo que se podría pensar en un primer momento, la orientación al mercado interno (OMI) no es contraria a la orientación al mercado externo sino todo lo contrario. La OMI permite que la organización derive a una orientación simétrica (Piercy, 1995) en el sentido de que mantiene un balance entre el grado de 
orientación interna y externa (Pitt y Foreman, 1999) reforzando la efectividad de sus respuestas estratégicas y su habilidad para satisfacer a los clientes de manera que la organización vea aumentar los servicios que presta y en consecuencia se generen mayores rendimientos (Gounaris, 2006). Según esta lógica y en términos comparativos, es tiempo de que las organizaciones que quieran ser competitivas adopten una adecuada OMI. En consecuencia, responderán al mercado de manera más efectiva que aquellas que sólo centren su atención en sus clientes externos (Lings, 1999; Bansal et al., 2001). En definitiva, en la base del argumento está la idea de que para desarrollar con éxito la orientación al mercado, primero hay que adoptar con fuerza una OMI (Piercy, 1995). Las empresas tienen la oportunidad y el desafío de asumir el reto de orientarse a su mercado interno para facilitar y propiciar que sus innovaciones tengan buenos resultados en el mercado y por ende, asegurar su competitividad.

\section{BIBLIOGRAFÍA}

Ahmed, P. K., y Rafiq, M. (1993): “The scope of internal marketing: defining the boundary between marketing and human resource management", Journal of Marketing Management, 9 (3), pp: 219-232.

Ahmed, P. K., y Rafiq, M. (2002): Internal marketing - Tools and concepts for customer-focused management, Butterworth-Heinemann Publications: Oxford.

Álvarez, J. C. (2006): Dirección por implicación (DPI). El cambio estratégico para competir en la sociedad del conocimiento, Pirámide: Madrid.

Atuahene-Gima, K. (1996): "Differential potency of factors affecting innovation performance in manufacturing and services firms in Australia", Journal of Product Innovation Management, 13, pp: 35-52. 
Avlonitis, G., Papastathopoulou, P. y Gounaris, S. (2001): "An empirically - based typology for product innovativeness for new financial services: success and failure scenarios", The Journal of Product Innovation Management, 18, pp: 324-342.

Bansal, H. S., Mendelson, M. B., Sharma, B. (2001): "The impact of internal marketing activities on external marketing outcomes", Journal of Quality Management, 6 (1), pp: 61-76.

Blazevic, V. y Lievens, A. (2004): "Learning during the financial service innovation process. Antecedents and performance effects", Journal of Business Research, 57, pp: 374-391.

Bourner, T. (1996): "The research process: four steps to success", in Greenfield, T. (Ed), Research methods: guidance for postgraduates, Arnold: London.

Carvalho Vieira, J. M., De Magalhães Serra, E. y Varela González, J. A. (2004): "New services margin/high sucess discriminators", The Services Industries Journal, 24 (5), pp:91-101.

Cooper, R. G. y De Brentani, U. (1991): "New industrial financial services: What distinguishes the winners", Journal of Product Innovation Management, 8 (2), pp: 7590.

Cooper, R. G., Easingwood, C. J., Edgett, S., Kleinschmidt, E. J. y Storey, C. (1994): "What distinguishes top performing new products in financial services", Journal of Product Innovation Management, 11, pp: 281-299.

Cuadrado Roura, J. R. (2004): "Tres preguntas en torno a los servicios: crecimiento, empleo, productividad", Papeles de Economía Española, 100 (2), pp: 211-237.

Czarnitzki, D. y Spielkamp, A. (2003): "Business services in Germany: Bridges for innovation": The Service Industries Journal, 23(2), pp: 1-30. 
De Brentani, U. (1995): "New industrial service development: Scenarios for success and failure", Journal of Business Research, 32, pp: 93-103.

De Brentani, U. y Ragot, E. (1996): "Developing new business to business professional services: what factors impact performance?", Industrial Marketing Management, 25, pp: 517-530.

Edgett, S. y Parkinson, S. (1994): "The development of new financial services. Identifying determinants of success and failure", International Journal of Service Industry Management, 5(4), pp: 24-38.

Eurostat (2007): http://epp.eurostat.ec.europa.eu

Gadrey, J., Gallouj, F. y Weinstein, O. (1995): "New modes of innovation - How services benefit industry", International Journal of Service Industry Management, 6 (3), pp: 4-16.

Gallouj, F. y Weinstein, O. (1997): "Innovation in services", Research Policy, 26, pp: 537-556.

George, W. G. y Marshall, C. E. (1984): Developing New Services. American Marketing Association, Proceedings Series.

Gounaris, S. P. (2006): "Internal market orientation and its measurement", Journal of Business Research, 59, pp: 432-448.

Johne, A. y Harborne, P. (2003): "One leader is not enough for major new service development: Results of a consumer banking study", The Service Industries Journal, 23 (3), pp: 22-39.

Johnson, S. P., Menor, L. J., Roth, A. V. y Chase, R.B. (2000): "A critical evaluation of the new service development process", in: Fitzsimons, J. y Fitzsimons M. (Eds.), New Service Development. Sage Publications, Thousand Oaks. 
Kolhi, A. y Jaworski, B. J. (1990): "Market orientation: the construct, research propositions, and managerial implications", Journal of Marketing, 54(2), pp:1-18

Lievens, A. y Moenaert, R. (2000a): "New service teams as information-processing systems. Reducing innovative uncertainty", Journal of Service Research, 3 (1), pp: 46-65.

Lievens, A. y Moenaert, R. (2000b): "Project team communication in financial service innovation", Journal of Management Studies, 37(5), julio, pp: 733-766.

Lievens, A., Moenaert, R. y S'Jegers, R. (1999): "Linking communication to innovation success in the financial services industry: a case study analysis", International Journal of Service Industry Management, 10 (1), pp: 23-47.

Lings, I. N. (1999): "Balancing internal and external orientation", Journal of Marketing Management, 15, pp: 239-263.

Lovelock, C. H. (1992): Managing Services: marketing, operations, and human resources, $2^{\mathrm{a}}$ Edición: Prentice Hall.

Martin, C. y Horne, D. (1993): "Services innovation: Successful versus unsuccessful firms", International Journal of Service Industry Management, 4 (1), pp: 49-65.

Martin, C. y Horne, D. (1995): "Level of success inputs for service innovations in the same firm", International Journal of Service Industry Management, 6 (4), pp: 40-56.

Matear, S., Gray, B. y Garrett, T. (2004): "Market orientation, brand investment, new service development, market position and performance for service organisations", International Journal of Service Industry Management, 15 (3-4), pp: 284-301.

Muller, E. y Zenker, A. (2001): "Business services as actors of knowledge transformation: the role of KIBS in regional and national innovation systems", Research Policy, 30, pp: 1501-1516. 
Narver, J. C. y Slater, S. F. (1990): "The effect of a market orientation on business profitability", Journal of Marketing, 54 (4), pp: 20-35

Penrose, E.T. (1959): The theory of the growth of the firm, Wiley: New York.

Piercy, N. (1995): "Customer satisfaction and the internal market: marketing our customers to our employees", Journal of Marketing Practice and Applied Marketing Science, 1 (1), pp: 22-44.

Pitt, L. F. y Foreman, S. K. (1999): "Internal marketing role in organizations: a transaction cost perspective", Journal of Business Research, 44, pp: 25-36.

Storey, C. y Easingwood, C. (1993): "The impact of the new product development project on the success of financial services", The Service Industries Journal, 13 (3), pp: 40-54.

Storey, C. y Easingwood, C. (1996): "Determinants of new product performance: a study in the financial services sector", International Journal of Service Industry Management, 7 (1), pp: 32-55.

Storey, C. y Easingwood, C. (1998): "The augmented service offering: A conceptualisation and study of its impact on new services success". Journal of Product Innovation Management, 15, pp: 335-351.

Van Riel, A. y Lievens, A. (2004): "New service development in high tech sectors: a decision making perspective", International Journal of service Industry Management, 15 (1), pp: 72-101.

\section{Cómo citar este artículo:}

Sánchez Hernández, Mª Isabel - Miranda González, Francisco J. ES LA HORA DEL MARKETING INTERNO.

REDMARKA - CIECID - Unidad de Investigación en Marketing Aplicado-Universidad de A Coruña, Número 2, V1, pp.37-53

ISSN 1852-2300

URL del Documento : http://www.cienciared.com.ar/ra/doc.php?n=1094 